\title{
NOUVEAUX TÉMOINS MANUSCRITS DE LA CHAÎNE DE POLYCHRONIOS SUR LE CANTIQUE (CPG C 83)
}

\author{
REINHART CEULEMANS / LEUVEN
}

Pour chacun des trois livres salomoniques, c'est-à-dire le Cantique des Cantiques ( $C P G$ C 83), les Proverbes ( $C P G$ C 90) et l'Ecclésiaste ( $C P G$ $C$ 102), la tradition manuscrite a transmis une chaîne exégétique grecque sous le nom de Polychronios le Diacre. ${ }^{1}$ Dans ces trois cas, ces chaînes étaient assez populaires, comme le montre le nombre assez élevé des manuscrits dans lesquels elles sont contenues. ${ }^{2}$ Malheureusement, aucune de ces trois chaînes n'est éditée de façon critique. On ne compte même aucune édition complète. ${ }^{3}$

Nous travaillons en tant que chargé de recherches du Fonds de la Recherche Scientifique - Flandre (FWO) à la Katholieke Universiteit Leuven. Pour cet article, la plupart des recherches ont été effectuées pendant un séjour en mai et juin 2010 à la Section grecque de l'Institut de recherche et d'histoire des textes (Paris), avec le support du FWO. Nous tenons à remercier le Dr. Paul Géhin et son équipe pour l'hospitalité chaleureuse qu'ils nous ont accordée.

1 Cette attribution est bien problématique. Si ces trois chaînes étaient en fait compilées par ce Polychronios et si les attributions des scholies dans ces trois chaînes sont exactes, cet auteur ne peut pas être identique à son homonyme d'Apamée, qui a rédigé un commentaire sur le livre de Job (CPG 3880), parce que plusieurs des scholies qui se trouvent dans ces trois chaînes appartiennent à des auteurs (comme Olympiodore le Diacre) qui sont postérieurs à Polychronios d'Apamée. NB: Dans cet article, nous utilisons le nom de «la chaîne de Polychronios» au lieu de «la chaîne du type I» ou «la chaîne du type D» (sur ces caractérisations, voir notre note 7). (Nous suivons la même argumentation en ce qui concerne la chaîne dite d'Eusèbe [ $C P G \mathrm{C}$ 84], dont nous faisons mention cidessous.)

2 En comparaison avec le nombre des manuscrits conservés des autres chaînes sur les livres salomoniques, celui des trois chaînes de Polychronios est assez fort. Voir en outre notre note 9. Avec P. Géhin, on pourrait appeler cette chaîne celle 'du type vulgate': voir Évagre le Pontique, Scholies aux Proverbes. Introduction, texte critique, traduction, notes, appendices et index par P. GÉHIN. SC, 340. Paris 1987, 70: «Le type I [= la chaîne de Polychronios sur les Proverbes] est celui qui est le plus abondamment représenté, en quelque sorte la vulgate, mais c'est aussi le type où les textes sont le plus abîmés».

3 Le seul texte imprimé complet de ces trois chaînes est une traduction latine de la chaîne sur les Proverbes, préparée au début du $17^{\mathrm{e}}$ siècle par Theodorus Peltanus: voir la référence dans la $C P G(\mathrm{C} 90)$ et les notices brèves de M. FAuLHaber, Hohelied-, Proverbien- und Prediger-Catenen. Theologische Studien der Leo-Gesellschaft, 4. Wien 1902, 122-123 et de L. Bossina, Note di storia della filologia patristica e di ecdotica catenaria: André Schott e l'infelice versione latina del commento In Canticum di Psello. Quaderni del Dipartimento di Fi- 
Seule une édition de la chaîne sur le Cantique a été publiée, en l'occurrence par Johannes van Meurs (Iohannes Meursius) en 1617. ${ }^{4}$ Cette édition non-critique n'est d'ailleurs pas complète: la partie avec les scholies sur Ct 4,4-7,5 y brille par son absence. À Louvain-la-Neuve, Jean-Marie Auwers prépare une édition critique; il a aussi déjà publié une liste électronique de tous les manuscrits connus de la chaîne de Polychronios sur le Cantique. ${ }^{5}$ Cette liste remplace celles composées en 1902 par Michael Faulhaber d'une part, et par Georg Karo et Hans Lietzmann, d'autre part. ${ }^{6}$ Ces savants allemands furent les premiers à identifier les différents types des chaînes sur les livres solomoniques et à classer les manuscrits connus. ${ }^{7}$ Depuis leurs publications, la chaîne de

lologia, Linguistica e Tradizione classica A. Rostagni 14 (2000) 257-258 (repris dans son Teodoreto restituito. Ricerche sulla catena dei Tre Padri e la sua tradizione. Studi e Ricerche, 68. Alessandria 2008, 199-201). La source manuscrite de cette traduction, que Peltanus n'avait pas identifiée, est inconnue, comme l'écrivent G. Karo/I. Lietzmann, Catenarum graecarum catalogus. Nachrichten von der Königlichen Gesellschaft der Wissenschaften zu Göttingen. Philologischhistorische Klasse (1902) 299. La CPG (C 83) fait aussi mention d'une traduction latine de la chaîne de Polychronios sur le Cantique, mais cette identification est incorrecte: ce texte latin est en fait une traduction de la chaîne dite d'Eusèbe (CPG C 84), et pas de celle de Polychronios. Voir E. Schulz-Flügel, Gregorius Eliberritanus, Epithalamium sive explanatio in Canticis Canticorum. Vetus Latina. Die Reste der altlateinischen Bibel, 26. Freiburg 1994, 110 note 1. Une traduction latine de la chaîne de Polychronios sur le Cantique n'existe pas en forme manuscrite.

4 Eusebii, Polychronii, Pselli in Canticum canticorum expositiones graecae. I. Meursius primus nunc e tenebris eruit, et publicavit. Lugduni Batavorum 1617, 75-112 (repris en 1746: voir la référence dans la CPG C 83). La seule source manuscrite de cette édition est identifiée par A. Rahlfs comme le Leidensis, Vulcanius 50 (s. XV-XVI), f. 48-69 $9^{\mathrm{v}}$. Voir A. RAHLFs, Die Catenenhandschrift des Meursius. Theologische Literaturzeitung 38 (1913) 763-764. Cf. aussi notre note 76 .

5 Voir son site web http://www.uclouvain.be/367267.html. Auwers a déjà achevé l'édition critique d'une autre chaîne (ou plutôt: d'un épitomé) sur le Cantique: celle de Procope de Gaza (CPG 7431): voir notre note 77. En outre, il prépare l'édition d'une troisième chaîne sur ce livre: celle dite d'Eusèbe.

6 Voir les références dans la note 3. Quelques ans avant la publication jointe de Karo/Lietzmann, ce dernier avait déjà identifié quelques types des chaînes sur le Cantique dans une étude préliminaire: H. Lietzmann, mit einem Beitrag von H. Usener, Catenen. Mitteilungen über ihre Geschichte und handschriftliche Überlieferung. Freiburg/Leipzig/Tübingen 1897, 57-65.

7 Karo/Lietzmann (cité note 3) ont dénommé la chaîne de Polychronius sur le Cantique la chaîne du 'type I' - dans le classement de FAUlHABER (cité note 3), elle est le 'type D'. 
Polychronios sur le Cantique n'est plus étudiée que très rarement. ${ }^{8}$ Par le biais de cette contribution, nous désirons contribuer au progrès de l'édition critique de cette chaîne, en mentionnant quelques témoins manuscrits inconnus. ${ }^{9}$

Grâce aux deux listes qui sont parues en 1902, on connaissait 13 témoins manuscrits de la chaîne de Polychronios sur le Cantique, dont 10 énumérés par Faulhaber et 9 par Karo/LietzManN: $:^{10}$

1. Mediolanensis, Ambrosianus A 148 inf (s. X-XI), f. $92^{v}-111^{\mathrm{v}}$

2. Venetus, Marcianus gr. 21 (s. XI), f. 106-123

3. Genuensis, Urbanus 2 (a. 1075), f. 119-148 (pas mentionné par Faulhaber)

4. Oxoniensis, Bodleianus, Laudianus gr. 30 A (s. XII), f. 96-114 $4^{11}$

5. Oxoniensis, Bodleianus, Auctarii E.2.16 (Misc. 44) (s. XII-XIII), f. $188-199^{\mathrm{v} 12}$

8 Les seuls chercheurs qui se sont penchés sur les sources et les méthodes de cette chaîne et sur ses relations avec les autres compilations sur le Cantique sont Faulhaber (son étude reste fondamentale), Auwers et M. Barbàra. Voir Faulhaber (cité note 3) 39-50; J.-M. Auwers, Chronique louvaniste: thèse d'agrégation de l'enseignement supérieur. Revue théologique de Louvain 39 (2008) 155; Origene, Commentario al Cantico dei Cantici. Testi in lingua greca. Introduzione, testo, traduzione e commento a cura di M. A. BARBÀrA. Biblioteca patristica, 42. Bologna 2005, 122-126.

9 Nous signalons que d'après le catalogue de G. de Andrés, les manuscrits perdus suivants semblent avoir contenu la chaîne de Polychronios sur le Cantique:

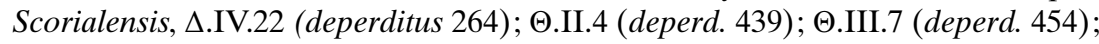

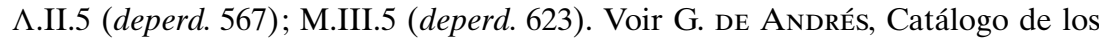
códices griegos desaparecidos de la Real Biblioteca de El Escorial. El Escorial 1968, ad loc. D'après lui, quelques-uns de ces manuscrits (le $\Theta . I I .4$ et peut-être le M.III.5) sont écrits par Andréas Darmarios, qui a copié plusieurs autres manuscrits de la chaîne de Polychronios sur le Cantique (comme le Basil. A.VII.6; le Monacensis gr. 294; l'Oxon. Auct. E.2.16; les Parisini gr. 173 et 948).

10 Faulhaber (cité note 3) 39-44 et Karo/LietzMann (cité note 3) 313-314 (plus tôt, Lietzmann [cité note 6] 57 avait déjà identifié le Parisinus gr. 151 comme témoin de la chaîne de Polychronios sur le Cantique). En grande partie, le bilan fourni par A. RAHLFs (Verzeichnis der griechischen Handschriften des Alten Testaments. Mitteilungen des Septuaginta-Unternehmens, 2. Berlin 1914, 415420) est fondé sur ceux de Faulhaber et Karo/Lietzmann, bien que lui-même y ait ajouté quelques autres témoins (voir ci-dessous).

11 D'après Karo/Lietzmann (cité note 3) 314, ce manuscrit est un des deux témoins (l'autre est l'Oxoniensis, Bodleianus, Auctarii E.2.16: voir la note suivante) qui ne fournissent que des extraits de la chaîne. Une telle description ne se trouve pas dans Faulhaber (cité note 3) 40-41, bien qu'il ait observé que deux scholies y manquent.

12 Il faut remarquer trois choses. (1) La numération des folios donnée par Faulhaber est différente de celle qu'on trouve aujourd'hui dans le manuscrit et dans 
6. Parisinus gr. 151 (s. XIII), f. $100^{\mathrm{v}}-116^{\mathrm{v}}$

7. Parisinus, Coislinianus 194 (s. XIII), f. $93^{\vee}-117$ (pas mentionné par FAulhaber)

8. Taurinensis, Bibliothecae Nationalis B.II.8 (Pasinus 97) (a. 1583), f. 22-42 (pas mentionné par Karo/Lietzmann)

9. Basilensis, Bibliothecae Universitatis A.VII.6 (gr. 15) (s. XVI), f. 89162 (pas mentionné par Faulhaber)

10. Monacensis gr. 294 (s. XVI), 73 f. (pas mentionné par KARo/LIETZMANN)

11. Romanus, Angelicus gr. 113 (s. XVI), f. $61^{\mathrm{v}}-75^{\mathrm{v}}$

12. Oxoniensis, Bodleianus, Auctarii E.2.17 (Misc. 45) (s. XVI-XVII), f. 1-32 (pas mentionné par Karo/LIETZManN)

13. Vaticanus gr. 1521 (s. XVI-XVII), f. 200-247 (pas mentionné par KARo/LiETZMANN).

En 1914, Alfred Rahlfs a identifié 3 autres témoins: $:^{13}$

14. Leidensis, Vulcanius 50 (s. XV-XVI), f. $48-69^{\mathrm{v} 14}$

les études postérieures: f. $186-197^{\mathrm{V}}$ chez FaulHaber (cité note 3) 41 sont f. $188-$ $199^{\mathrm{v}}$ dans le numérotage actuel du manuscrit. (2) Ce manuscrit contient en fait deux chaînes sur le Cantique: la chaîne de Polychronios (qui n'est pas tout à fait complète: voir notre remarque suivante) aux f. $188-199^{\mathrm{v}}$ et une chaîne anonyme aux f. $182-187^{\mathrm{v}}$ (qui n'interprète que les deux premiers chapitres du Cantique). FAUlHABER (cité note 3) 41 a décrit ce texte comme «eine eigene Erklärung zum hohen Liede $1^{1}-2^{17}$ ». Pareillement, RAHLFs, Verzeichnis (cité note 10) 164 et 424 et U. et D. Hagedorn (Die älteren griechischen Katenen zum Buch Hiob. Band I. Einleitung, Prologe und Epiloge, Fragmente zu Hiob 1,1-8,22. PTS, 40. Berlin/New York 1994, 10) l'ont nommé un commentaire sur Ct 1-2. Toutefois, BARBÀra, Origene (cité note 8) 132 note 127 l'a décrit comme une chaîne anonyme autonome, qui contient des fragments anonymes de Philon de Carpasie ( $C P G$ 3810), Grégoire de Nysse ( $C P G$ 3158) et Théodoret de Cyr (CPG 6203). Ces scholies sont contaminées et ne se laissent identifier que difficilement. On attend une étude et édition de ce texte. (3) Les f. $188-199^{\mathrm{v}}$ de ce manuscrit ne fournissent le texte de la chaîne de Polychronios que dès $\mathrm{Ct}$ 1,5. En outre, dans sa liste électronique (voir l'adresse que nous mentionnons plus haut, note 5), Auwers a décrit ce manuscrit comme témoin d'une rédaction abrégée. Déjà Karo/Lietzmann (cité note 3) 314 avaient remarqué que ce manuscrit est un témoin qui ne contient que des extraits de la chaîne et qui omet quelques scholies dans le quatrième chapitre. Une observation similaire était faite par FAulhaber (cité note 3) 41, alors qu'il a ajouté que, vers la fin du texte, la rédaction n'était plus abrégée. Cette conclusion est aussi tirée par BARBÀRA, Origene (cité note 8) 124-125 et note 172.

13 RAhlFs, Verzeichnis (cité note 10) 95-96, 205 et 208-209. 
15. Parisinus gr. 173 (s. XVI), f. $1-89^{\mathrm{v} 15}$
16. Parisinus gr. 948 (s. XVI), f. $45-127 .{ }^{16}$

Enfin, a cette liste de 16 témoins, Auwers l'a encore accrue de 2 témoins additionnels $:^{17}$

17. Cantabrigiensis, Collegii Trinitatis B.7.3. (s. XIII), f. $213-236^{18}$

18. Berolinensis, Phillippicus 1412 (ca. a. 1540), f. $132^{\mathrm{v}}-156$.

Ce total de 18 manuscrits connus de la chaîne de Polychronios figure sur le site web d'Auwers (dont nous avons donné l'URL dans notre note 5).

Désormais nous pouvons y ajouter quatre autres témoins complets (no. 19-22), dont deux ont été identifiés par nous-mêmes. L'identifi-

14 En fait, ce manuscrit avait déjà été signalé par Rahlfs une année plus tôt, quand il l'avait identifié comme la source de l'édition de Meursius. Voir la référence dans notre note 4.

15 Voir la note suivante.

16 Il faut signaler deux choses. (1) L'identification faite par Rahlfs des deux manuscrits parisiens (173 et 948) n'était pas sûre, comme le montre le point d'interrogation qu'il avait ajouté (voir RAHLFs, Verzeichnis [cité note 10] 205 et 208 209). Elle est confirmée par Auwers, qui a inclus ces deux manuscrits dans sa liste électronique (voir la référence dans notre note 5). (2) La chaîne de Polychronios n'est pas le seul texte que ces deux manuscrits parisiens ont en commun. Ils contiennent aussi un autre texte, dont Rahlfs a suggéré qu'il était celui de la chaîne du Ps.-Eusèbe sur le Cantique (mentionnée plus haut). Cette identification doit être rejetée: récemment, Auwers a qualifié ce texte comme étant un commentaire (et donc pas une chaîne) inconnu qui est attribué à Eusèbe de Césarée mais qui est en fait beaucoup plus jeune. Voir J.-M. Auwers, L'interprétation du Cantique des cantiques à travers les chaînes exégétiques grecques (Epitomé de Procope, chaîne de Polychronios, chaîne dite d'Eusèbe, Catena Barberiniana), Dissertation présentée pour l'obtention du grade d'Agrégé de l'Enseignement Supérieur. Louvain-la-Neuve 2007, I, 124 note 12 et II, 424 note 42 . Plus avant dans cet article, nous identifions un troisième témoin de ce commentaire, l'Oxoniensis, Bodleianus, Auctarii X.2.5.12 (D’Orville 414), qui est certainement un apographon d'un de ces deux manuscrits parisiens.

17 Voir le site web auquel nous nous référons ci-dessus (note 5).

18 Précédemment, ce texte était caractérisé comme un commentaire d'un auteur inconnu (RAHLFS, Verzeichnis [cité note 10] 40 et 424) ou comme le sommaire d'un commentaire inconnu (Gregorii Nysseni in Canticum Canticorum. Edidit H. Langerbeck. Gregorii Nysseni opera, 6. Leiden 1986 [= 1960], LiI). Comme Auwers, Barbàra a identifié ce texte comme la chaîne de Polychronios, mais elle l'a décrit comme une rédaction abrégée. Voir BARBÀrA, Origene (cité note 8) 125 et C. Curti/M. A. Barbàra, Catene esegetiche greche, dans A. Di Berardino (éd.), Patrologia. Vol. V: Dal Concilio di Calcedonia (451) a Giovanni Damasceno $(\uparrow 750)$, I Padri orientali. Institutum Patristicum Augustinianum. Genova $2001[=2000], 633$. 
cation des deux autres est fournie dans les catalogues de leurs bibliothèques respectives; dans ce cas, nous n'avons pas vu le manuscrit en question et nous nous contentons de nous référer aux catalogues.

19. Mosquensis, Archives d'État russes des documents anciens, Fonds 1607, 1.22 (olim Dresdensis A 107) (s. XII), f. 37-55'

D'après la description que fournit le catalogue de Franz Schnorr von Carolsfeld, ce manuscrit en parchemin compte 55 feuillets et il mesure environ 28 sur $20 \mathrm{~cm}$. Il contient trois textes, qui étaient décrits sommairement par Schnorr von Carolsfeld comme les textes septantiques de l'Ecclésiaste, d'une partie des Proverbes et du Cantique, tous avec des scholies patristiques. ${ }^{19}$ Une telle description vague se retrouve aussi dans la célèbre édition de Robert Holmes et James Parsons,${ }^{20}$ chez Erich Klostermann ${ }^{21}$ et surtout chez Rahlfs, ${ }^{22}$ dont les renseignements brefs semblent dire que ce manuscrit ne contient que le texte de la Septante de ces trois livres et que seul celui des Proverbes est accompagné par des extraits caténaires.

En fait, ces trois textes sont tous des chaînes. La chaîne sur l'Ecclésiaste (f. $1-28^{v}$ : chaîne complète) a été récemment identifiée par Peter Gentry comme celle de Polychronios. ${ }^{23}$ De même la chaîne sur les

19 F. Schnorr von CAROLSFEld, Katalog der Handschriften der Sächsischen Landesbibliothek zu Dresden. Korrigierte und verbesserte, nach dem Exemplar der Landesbibliothek photomechanisch hergestellte Ausgabe. Dresden 1979, I, 46.

20 Vetus Testamentum Graecum cum variis lectionibus. Editionem a R. Holmes inchoatam continuavit J. PArsons. Oxonii 1823, III, Praefatio ad librum Proverbiorum (sans pagination): cote 159. Voir aussi la description qu'a donnée antérieurement G. L. Spohn, Der Prediger Salomo, aus dem hebräischen aufs neue übersetzt und mit kritischen Anmerkungen begleitet. Nebst einer Beylage, welche Varianten zu dem Prediger in den LXX. aus zweyen Manuskripten und dem Olympiodor enthält, Leipzig 1785, 111 (cote 2).

21 E. Klostermann, De libri Coheleth versione Alexandrina, Dissertatio Inauguralis. Kiel 1892, 6-7. C'est lui qui a remarqué que le Dresdensis A 107, le ms. 159 dans l'édition de Holmes/Parsons, et le ms. 2 de Spohn sont un seul et même manuscrit.

22 Rahlfs, Verzeichnis (cité note 10) 50.

23 P. J. Gentry, dans deux conférences données aux congrès SBL Annual Meeting (Boston, MA, 24/11/2008) et Von der Septuaginta zum Neuen Testament: Textgeschichtliche Erörterungen (Wuppertal, 30-31/03/2009). Que le Prof. Gentry (Louisville, KY), qui a bien voulu nous communiquer le texte de ses communications, trouve ici l'expression de notre gratitude. Le Mosq., Arch. nat. $\Phi$. 1607, 1.22 doit donc être ajouté aux listes des témoins de la chaîne de Polychronios sur l'Ecclésiaste composées par A. LABATE: voir ses articles Nuovi codici della catena sull'Ecclesiaste di Policronio. Augustinianum 18 (1978) 551- 
Proverbes (f. 29-36 : seulement Prov $25,1-28,12)^{24}$ et celle sur le Cantique (chaîne complète) sont-elles des chaînes de Polychronios, comme nous les avons identifiées nous-mêmes. ${ }^{25}$ En ce qui concerne la chaîne sur le Cantique, le manuscrit est un témoin d'une rédaction qui est un peu plus extensive que celle qui était éditée par Meursius et qui se trouve aussi dans le Mediolanensis, Ambrosianus A 148 inf et dans le Parisinus gr. 151.

Klostermann avait remarqué qu'initialement le Mosq., Arch. nat. $\Phi$. 1607, 1.22 faisait partie d'un autre codex, car il contient encore le numérotage antérieur des cahiers: le f. 1 , p.ex., était le début du cahier $1 \beta^{\prime}$. Par conséquent, il avait émis l'hypothèse que dans le codex original complet, les Proverbes (là aussi n'ayant leur début qu'au $25^{\mathrm{e}}$ chapitre) étaient précédés par Job. ${ }^{26}$ Quelques années plus tard, Otto von Gebhardt écrivait (apparemment indépendamment de Klostermann) que le codex, dont le Mosq., Arch. nat. Ф. 1607, 1.22 avait fait partie, était sans aucun doute le Mosquensis, Bibliothecae Synodalis 392 (Vladimir 42$).{ }^{27}$ Dans son état actuel, ce dernier manuscrit, qui mesure 280 à

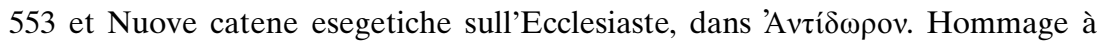
Maurits Geerard pour célébrer l'achèvement de la Clavis Patrum Graecorum. Wetteren 1984, I, 241-263.

24 De la chaîne sur les Proverbes, les seules photos que nous avons vues, sont celles des f. 36-36 . F. 36 s'ouvre in medias res, avec l'exégèse de Prov 27,25-26. F. $36^{\mathrm{V}}$ se termine avec l'exégèse de Prov 28,9-12. Le début de la part consacrée aux Proverbes était identifié par Holmes/Parsons (cité note 20) III, praef. Prov. comme Prov 25,1. À l'IRHT, nous avons eu l'occasion de confronter cette pièce de chaîne avec les transcriptions de plusieurs manuscrits des chaînes sur les Proverbes, faites par M. Richard et P. Géhin. Nous pouvons conclure que la chaîne sur les Proverbes du Mosq., Arch. nat. Ф. 1607, 1.22 est sans aucun doute celle de Polychronios.

25 Grâce à l'aide de Gentry, nous avons pu consulter des photos des f. $37-55^{\mathrm{v}}$ (chaîne sur le Cantique). Malheureusement, dans son état actuel, le Mosq., Arch. nat. $\Phi$. 1607, 1.22 est difficile à lire: il y a beaucoup de trous dans les feuillets et très souvent, le texte d'un folio est difficile à distinguer de celui des folios suivants et précédents qui a traversé le papier. Ici, nous ne décrirons pas ces dommages en détail, car ils sont le sujet de recherches qui sont en train d'être menées par F. Albrecht (Göttingen) et Gentry. Comme ce dernier nous l'a indiqué, la cause de ces dégâts est l'eau, qui a mouillé l'encre au gallo-tannate de fer.

26 E. Klostermann, Analecta zur Septuaginta, Hexapla und Patristik. Leipzig $1895,39$.

27 O. von Gebhardt, Christian Friedrich Matthaei und seine Sammlung griechischer Handschriften. Centralblatt für Bibliothekswesen 15 (1898) 477. 
$199 \mathrm{~mm}$ et compte 218 feuillets de parchemin ${ }^{28}$ contient une chaîne incomplète sur Prov 1,1-31,31 (aux f. 1-84, identifiée par Karo/ Lietzmann comme la chaîne de Polychronios) $)^{29}$ et une chaîne sur Job 1,1-42,10 (aux f. 85-218, identifiée par Karo/Lietzmann comme celle de Polychronios).$^{30}$ D'après von Gebhardt, entre les f. $84^{31}$ et 85 de ce manuscrit, il manque les cahiers $\imath \beta^{\prime}-1 \eta$ ' et le début du $\imath \theta^{\prime}$ ': ces cahiers sont le Mosq., Arch. nat. Ф. 1607, 1.22 dans son état actuel. ${ }^{32}$

Selon cette hypothèse, le codex originel a contenu la chaîne de Polychronios sur les trois livres salomoniques et Job, dans cet ordre: la chaîne sur Prov 1,1-31,31 (sans Prov 25-29); sur l'Ecclésiaste; sur Prov 25,1-28,12; sur le Cantique; sur Job $1,1-42,10 .{ }^{33}$ Cet ordre (c'est-à-dire: Prov - Eccl - Ct - Job) est tout à fait normal: on le retrouve dans tous les manuscrits connus (à l'exception d'un seul) qui contiennent la chaîne de Polychronios sur ces quatre livres sapientiaux. ${ }^{34}$ À cet égard donc,

28 Voir: l'Archimandrite Vladimir, Sistematičeskoe opisanie rukopisej Moskovskoj Sinodal'noj (Patriaršej) Biblioteki. I. Rukopisi grečeskija. Moskva 1894, 43 et B.L. Fonkič/F. B. Poljakov, Grečeskie rukopisi Moskovskoj Sinodal'noj biblioteki. Paleografičeskie, kodikologičeskie i bibliografičeskie dopolnenija $\mathrm{k}$ katalogu archimandrita Vladimira (Filantropova). Moskva 1993, 34. À l'IHRT, nous avons aussi consulté la traduction française du catalogue de Vladimir, faite par X. Grichine et révisée par J. Johannet, I, ${ }^{2} 1995,49$.

29 Karo/Lietzmann (cité note 3) 302. Rappelons-nous que dans la chaîne de Polychronios sur les Proverbes, l'ordre du texte biblique est le suivant (comme nous l'avons observé dans la transcription que M. Richard a faite du Paris. gr. 151): Prov 1,1-24,22; 30,1-14; 24,23-34; 30,15-31,9; 31,10-31; 25,1-29,27. Dans le Mosq., Syn. 392, la chaîne est complète jusqu'à Prov 31,31: les chapitres 25-29 manquent.

30 Karo/Lietzmann (cité note 3) 322. Les Hagedorn (cité note 12) I, 21, n'ayant pas vu le manuscrit, ont suivi le jugement de Karo/Lietzmann.

31 Dans son état actuel, il y a deux f. 84 dans le manuscrit. Ici il s'agit du deuxième f. 84.

32 Voir l'argumentation plus détaillée dans von Gebhardt (cité note 27) 477 (et son note 1). Quelques années plus tard, Karo/LiETzMann (cité note 3) 302 ont remarqué que le numérotage actuel des cahiers dans le Mosq. Syn. 392 provient d'une main postérieure. Malheureusement, le catalogue de VLADIMIR (cité note 28) ne contient aucun renseignement à ce sujet.

33 Contrairement à l'hypothèse de Klostermann, Analecta (cité note 26) 39 selon laquelle la chaîne sur l'Ecclésiaste serait précédée par celle sur Job (au lieu de celle sur Prov 1,1-31,31).

34 Le Mediolanensis, Ambrosianus A 148 inf; le Venetus, Marcianus gr. 21; l'Oxoniensis, Bodleianus, Laudianus gr. 30 A; le Parisinus gr. 151; le Parisinus, Coislinianus gr. 194; le Romanus, Angelicus gr. 113. La seule exception est l'Oxoniensis, Bodleianus, Auctarii E.2.16 (Misc.44), dans lequel la chaîne sur Job précède celles aux livres salomoniques. Dans ce manuscrit, l'ordre est interrompu par la présence d'une deuxième chaîne partielle sur le Cantique, précé- 
l'hypothèse de von Gebhardt est réellement pertinente. Néanmoins, la division de la chaîne sur les Proverbes en deux parts s'avère étrange, comme l'est aussi la présence de la chaîne sur l'Ecclésiaste entre elles. En outre, l'absence de la chaîne sur les derniers versets des Proverbes $(28,13-29,27)$ demeure inexpliquée.

La datation du $10^{\mathrm{e}}-11^{\mathrm{e}}$ siècle que Schnorr von Carolsfeld a assignée au Mosq., Arch. nat. Ф. 1607, 1.22 et qui est acceptée par Rahlfs, ${ }^{35}$ s'avère inconciliable avec l'hypothèse de von Gebhardt: le Mosq., Syn. 392 a été daté par Vladimir au $12^{\mathrm{e}}$ siècle. ${ }^{36}$ Plus récemment, Boris Fonkič et Fëdor Poljakov ont précisé cette datation, la fixant à la deuxième moitié du $12^{\mathrm{e}}$ siècle. ${ }^{37}$ Néanmoins, cette datation-là du Mosq., Arch. nat. Ф. 1607, 1.22 n'est pas si sûre: contre Schnorr von Carolsfeld et les autres savants cités plus haut, Oskar Lehmann a daté l'écriture de ce manuscrit du $12^{\mathrm{e}}$ ou du $13^{\mathrm{e}}$ siècle. ${ }^{38}$ Cette datation de Lehmann reçoit l'appui de Gentry, ${ }^{39}$ qui a fait observer que le type d'encre du manuscrit (sur celui-ci: voir notre note 25 ) n'a été utilisé que dès le $12^{\mathrm{e}}$ siècle. Cette datation concorde avec celle qu'on a proposé pour le Mosq., Syn. 392: cet appui est un argument en faveur de l'hypothèse de von Gebhardt.

Cette hypothèse, si elle est correcte, ${ }^{40}$ revêt une grande importance pour notre connaissance de l'histoire du Mosq., Arch. nat. $\Phi .1607,1.22$.

dant celle de Polychronios (voir notre note 12): à cet égard, ce manuscrit est unique.

35 Schnorr von Carolsfeld (cité note 19) I, 46 et Rahlfs, Verzeichnis (cité note 10) 50. Plus tôt, Spohn (cité note 20) 111 (suivant les indications de Chr. F. de Matthaei, sur lequel voir notre note 41) et Holmes/Parsons (cité note 20) III, praef. Prov. l'avaient daté du $11^{\mathrm{e}}$ siècle, suivis par Klostermann, De libri Coheleth (cité note 21) 6.

37 Fonkič/Poljakov (cité note 28) 34.

38 O. Lehmann, Die tachygraphischen Abkürzungen der griechischen Handschriften. Hildesheim 1965 [= 1880], 73, 82, 95.

39 Dans ses deux communications mentionnées plus haut (note 23).

40 Bien entendu, nous-mêmes, qui n'avons vu que les photos de quelques folios du Mosqu., Arch. nat. $\Phi$ 1607, 1.22 et aucune du Mosq., Syn. 392, ne sommes pas apte à confirmer ou à nier l'hypothèse de von Gebhardt. Plus haut, nous avons énuméré quelques observations pour et contre son argumentation, mais dans l'ensemble, elle nous semble convaincante. Comme pierre de touche additionnelle pour la contrôler, on pourrait examiner si, dans la tradition manuscrite, la position du Mosq. Bibl. Syn. 392 dans le stemma de la chaîne de Polychronios sur les Proverbes et sur Job est semblable à celle du Mosq., Arch. nat. $\Phi$. 1607, 1.22 dans le stemma de cette chaîne sur l'Ecclésiaste et sur le Cantique. Une telle 
Grâce aux renseignements fournis par Schnorr von Carolsfeld et Klostermann et surtout par Spohn et von Gebhardt, on peut déduire que le Mosq., Arch. nat. Ф. 1607, 1.22 est un des manuscrits que Christian Friedrich de Matthaei avait volés de la Bibliothèque synodale de Moscou et qu'il avait vendus à la bibliothèque de Dresde en $1788 .{ }^{41} \mathrm{En}$ 1947, ces manuscrits furent rendus à Moscou, où ils sont gardés dans le fonds 1607 des Archives d'État russes des documents anciens. ${ }^{42} \mathrm{Si}$ l'hypothèse de von Gebhardt est juste, et si le Mosq., Arch. nat. Ф. 1607, 1.22 faisait bien originalement partie du Mosq., Syn. 392, on peut retracer son histoire plus loin dans le passé. D'après la description qu'a fournie l'Archimandrite Vladimir pour le Mosq., Syn. 392, les f. 1-2 de ce manuscrit contiennent respectivement la signature d'Arsenji Suchanov et l'indication qu'il a appartenu au monastère de Philotheou

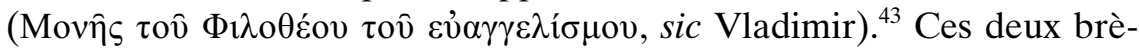
ves notices montrent que le Mosq., Syn. 392 (et donc aussi l'Arch. nat. $\Phi .1607,1.22)$ représente un des plus ou moins 450 manuscrits grecs qu'Arsenji Suchanov avait transportés des monastères athonites à la Russie en $1654-1655$. $^{44}$

étude (qui dépasse le but de cet article) est importante surtout pour les deux fragments de la chaîne sur les Proverbes.

41 Schnorr von Carolsfeld (cité note 19) I, 46 et Klostermann, De libri Coheleth (cité note 21) 7 ont indiqué que le manuscrit avait été décrit par de Matthaei. SpoHn (cité note 20) 109-111 a signalé explicitement que le manuscrit était en possession de de Matthaei. Dans son article (cité note 27), von GEBHARDT a cité le manuscrit comme un de ceux qui avaient été volés par de Matthaei. Bien que ce manuscrit ne figure pas dans les catalogues imprimés de de Matthaei, le Mosq., Arch. nat. $\Phi .1607,1.22$ peut être trouvé (cote: 15) dans la liste manuscrite qu'il a donnée à la bibliothèque de Dresde et qui énumère les manuscrits qu'il a vendus (cf. SCHNORR vON CAROLSFELd [cité note 19] I, XI). En outre, c'est de Matthaei qui a collationné ce manuscrit pour l'édition de HoLmes/Parsons (cité note 20). Voir Klostermann, Analecta (cité note 26) 39.

42 Voir les renseignements fournis par J.-M. OLIVIER, Répertoire des bibliothèques et des catalogues de manuscrits grecs de Marcel Richard. Corpus Christianorum. Turnhout ${ }^{3} 1995,570$.

43 Vladimir (cité note 28) I, 43.

44 Voir P. Lemerle, La vie ancienne de saint Athanase l'Athonite composée au début du XI ${ }^{\mathrm{e}}$ siècle par Athanase de Lavra, dans Le millénaire du Mont Athos 963-1963. Études et Mélanges. Chevetogne 1963, I, 65 note 14 et la littérature citée là-bas; Vitae duae antiquae Sancti Athanasii Athonitae editae a J. Noret. CCSG, 9. Tunhout/Leuven 1982, xxx-XxxI. 
20. Matritensis, Bibliothecae March 1 (a. 1579), f. 65-116

En 1974, Gregorio de Andrés a donné la description des deux manuscrits grecs qui se trouvent dans la collection privée de don Bartolomé March à Madrid..$^{45}$ Le premier de ces manuscrits, écrit en 1579 par Andréas Darmarios, contient trois textes: les chaînes complètes de Polychronios sur l'Ecclésiaste (f. 1-64) et sur le Cantique; et l'In Canticum de Michel Psellos (f. $117-160^{v}$ ). ${ }^{46}$

\section{Novi Portensis, Yalensis Beineckianus 273 (olim Caesaraugustensis,} Pilar 1587) (a. 1587), f. 1-53'

D'après le catalogue préparé par Barbara Shailor, ce manuscrit, qui se compose en fait de deux codices, contient le texte de la chaîne de Polychronios sur le Cantique et sur l'Ecclésiaste (f. 55-118v). Chacun de ces manuscrits est achevé par Andréas Darmarios en $1587 .{ }^{47}$

\section{Berolinensis, Phillippicus 1484 (s. XVII), f. $82^{\mathrm{v}}-111$}

Ce manuscrit compte 190 feuillets et s'ouvre avec une chaîne sur les Odes, sur l'Ecclésiaste et sur le Cantique. Elles sont suivies par un amalgame de textes dogmatiques et ascétiques. ${ }^{48}$ Dans leur catalogue, Wilhelm Studemund et Leopold Cohn décrivent le texte des f. $82^{\mathrm{v}}-111$ comme le Cantique accompagné par un commentaire anonyme. ${ }^{49}$ Nous pouvons fournir une identification plus précise: ces feuillets contiennent, sous forme d'une chaîne à pleine page, la chaîne de Polychronios

45 G. DE Andrés, Catálogo de los códices griegos de las colecciones: Complutense, Lázaro Galdiano y March de Madrid. Cuadernos de filología clásica 6 (1974) $262-265$.

46 Ce témoin n'est connu ni par Labate, qui s'est engagé à préparer une édition de la chaîne de Polychronios sur l'Ecclésiaste, ni par L. Westerink, qui a préparé l'édition du texte psellien. Voir Labate, Nuovi codici (cité note 23) et Michaelis Pselli Poemata, recensuit L. G. Westerink. Bibliotheca Scriptorum Graecorum et Romanorum Teubneriana. Stutgardiae/Lipsiae 1992, X-XII.

47 B. A. Shailor, Catalogue of Medieval and Renaissance Manuscripts in the Beinecke Rare Book and Manuscript Library, Yale University. Volume II: mss 251-500. Medieval \& Renaissance Texts and Studies, 48. Binghamton, NY 1987, 40-41. Voir aussi A. Karpozilos, The Yale University Manuscripts of Andreas Darmarius. Hellenika 26 (1973) 69.

48 Voir le catalogue auquel nous nous référons dans la note suivante et F. DiEKAMP, Analecta Patristica. Texte und Abhandlungen zur griechischen Patristik. OCA, 117. Roma 1938, 184.

49 Codices ex Bibliotheca Meermanniana Phillippici graeci nunc Berolinenses descripserunt G. Studemund/L. Cohn. Berolini 1890, 32-33 (cote: 80). Observez que le numérotage du manuscrit que nous fournissons est différent de celui du catalogue. 
sur le Cantique. À première vue, sa rédaction nous semble très proche de celle qu'on trouve dans le Genuensis, Urbanus 2 (a. 1075). La raison pour laquelle son identification est défectueuse dans le catalogue allemand ressort clairement: dans le manuscrit, la chaîne omet le titre. Pour le reste, le texte s'y trouve au complet.

D'après Studemund et Cohn, le manuscrit avait été écrit au $17^{\mathrm{e}}$ siècle par Jacques Sirmond. Néanmoins, au f. 111 (qui n'est pas le dernier folio du manuscrit), la dernière scholie de la chaîne sur le Cantique est suivie par un colophon qui identifie le copiste comme Théodore le moine

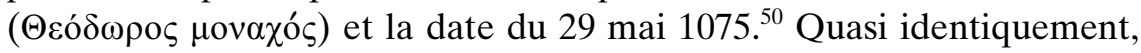
ce colophon se retrouve à la fin de la chaîne de Polychronios sur le Cantique dans le Genuensis, Urbanus 2 (mentionné plus haut dans cet article). ${ }^{51}$ Bien qu'une telle hypothèse doive être vérifiée sur la base d'une collation complète et détaillée, il nous semble très probable que le texte de la chaîne de Polychronios sur le Cantique du Phillippicus 1484 soit copié sur base de l'Urbanus $2 .^{52}$

Ces quatre manuscrits (= no. 19-22) doivent donc être ajoutés aux dixhuit témoins énumérés plus haut.

En outre, il existe quelques manuscrits qui attestent partiellement ou indirectement de la chaîne de Polychronios sur le Cantique. Ils ne contiennent qu'une partie du texte et/ou ils témoignent de compilations dérivées de celle de Polychronios ou ayant pris cette chaîne comme une de leurs sources. Comme aucun de ces manuscrits n'est mentionné dans la liste d'Auwers, ${ }^{53}$ il nous semble utile de les signaler ici.

Un tel manuscrit avait déjà été identifié par Maria Barbàra:

50 Le colophon est repris dans Studemund/Cohn (cité note 49) 32.

51 Voir A. Cataldi Palau, Catalogo dei manoscritti greci della Biblioteca Franzoniana (Genova) (Urbani 2-20). Supplementi al Bollettino dei Classici, 8. Roma 1990, 35-38 (le colophon est cité à la p. 36). Rappelons-nous que ce manuscrit génois, qui compte 148 feuillets tous écrits par une seule main, contient trois textes: la chaîne de Polychronios sur les Proverbes (f. $1-85^{\mathrm{v}}$ ), sur l'Ecclésiaste (f. 86-119) et sur le Cantique (f. $\left.119^{\mathrm{v}}-148\right)$. Le colophon se trouve donc à la fin du manuscrit.

52 Comme les traditions des chaînes sur les trois livres salomoniques sont très semblables, il est bien possible aussi qu'en ce qui concerne la chaîne de Polychronios sur l'Ecclésiaste, le Phillippicus 1484 soit une copie de l'Urbanus $K$ Malheureusement, la nature de ce texte dans ces deux manuscrits n'est jamais étudiée: Labate ne les a pas vus (voir son Nuovi codici [cité note 23] 553).

53 Voir le site web auquel nous nous référons supra (note 5). 
23. Sinaiticus gr. 311 (a. 1510), f. 59-71

D'après Barbàra, d'une part ce texte représente une rédaction abrégée de la chaîne de Polychronios sur le Cantique, mais d'autre part il contient d'autres scholies. ${ }^{54}$ Nous ne l'avons pas vu.

Nous pouvons y ajouter deux autres:

\section{Romanus, Collegii graeci 16 (s. XIV), f. $70^{\mathrm{v}}-108$ [=109]}

Les renseignements sur ce manuscrit que fournit Spiridon Lambros dans son catalogue sont très brefs. ${ }^{55}$ On peut en déduire (a) que les $\mathrm{f}$. $70^{v}-108$ [= 109] contiennent le texte septantique du Cantique, qui est accompagné par des morceaux d'exégèse et (b) que cette exégèse se compose surtout - mais pas exclusivement - de l'In Canticum de Michel Psellos. Nous avons identifié cette exégèse plus précisément. ${ }^{56}$

La chaîne s'ouvre avec les prologues $1 \mathrm{a}$ et $1 \mathrm{~b}$ de la chaîne de Polychronios (sur ceux-ci, voir ci-dessous). Cette introduction est accompagnée par deux scholies marginales qui résument des scholies de la catena Hauniensis. ${ }^{57}$ De Ct 1,1 à 7,1 (f. 70-103 [=104]), le manuscrit contient une chaîne se composant de deux sources, dont chacune d'entre elles se retrouve dans son état complet: l'In Canticum de

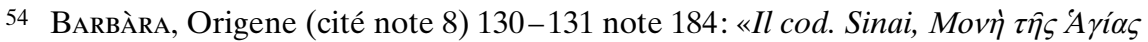

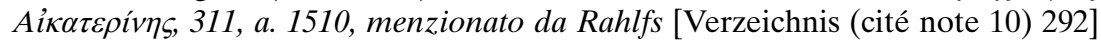
come testimone di un Comm. in Cant., tramanda la catena di Policronio; ne riporta una redazione abbreviata ancor più che il cod. Cantabrigense, Trinity Coll. gr. B. 7. 3 [sur laquelle, voir notre note 18], ma anche con scolii là omessi e peraltro noti». Voir aussi Curti/Barbàra (cité note 18) 633. Les descriptions fournies dans les catalogues de GardTHAUSEN et de KAMIL sont trop sommaires pour être utiles. Voir V. Gardthausen, Catalogus codicum graecorum sinaiti-

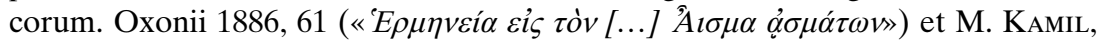
Catalogue of All Manuscripts in the Monastery of St. Catherine on Mount Sinai. Wiesbaden 1970, 74 (cote 359: «Commentary on [...] Song of Solomon»).

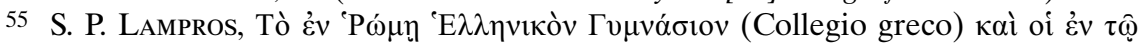

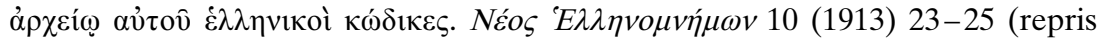
dans Catalogi codicum Graecorum qui in minoribus bibliothecis Italicis asservantur in duo volumina collati et novissimis additamentis aucti. Accuravit et indices adiecit $\mathrm{CH}$. SAmberger. Catalogi codicum Graecorum lucis ope reimpressi. Lipsiae 1968, II, 275-277).

56 Grâce à P. Gentry, nous avons pu voir les photos d'une grande partie du manuscrit.

57 Sur cette chaîne, ignorée par Faulhaber (cité note 3) et Karo/Lietzmann (cité note 3), voir R. Ceulemans, A Catena Hauniensis Discovered for the Book of Canticles. Ephemerides Theologicae Lovanienses 85 (2009) 63-70. 
Psellos $^{58}$ et la chaîne de Polychronios. Donc, jusqu'a Ct 7,1 le Rom., Coll. gr. 16 est un témoin du texte complet de la chaîne de Polychronios. Les noms d'auteurs ne sont jamais donnés, à l'exception de ceux de Polychronios et de Psellos (f. 72). Il est clair que la chaîne de Polychronios représente une des deux sources utilisées par le compilateur: les scholies différentes qui, dans cette chaîne, suivent le même кsí $\mu \varepsilon v o v$, sont ici présentées comme une seule scholie.

De Ct 7,2 à 8,14 (f. 103-108 [= 104-109], Ductus un peu différent), le manuscrit renferme une compilation qui se laisse percevoir comme un recueil de deux chaînes sur le Cantique. Le compilateur a choisi des extraits de la chaîne de Polychronios et de la catena Hauniensis et les a présentés ensemble. Parfois les extraits d'une chaîne sont séparés de ceux de l'autre par le mot ờ $\lambda \lambda \mathrm{o}$ (ou ع̈ $\varepsilon \varepsilon \rho o[s]$ ), mais le plus souvent, les uns se retrouvent mélangés aux autres. Autrement dit, chaque scholie comporte des éléments de la chaîne de Polychronios et de la catena Hauniensis; aucune de ces deux chaînes n'est complète. ${ }^{59}$ À une seule reprise, le nom d'un auteur est fourni (celui de Grégoire de Nysse, $\mathrm{f}$. $\left.107^{\mathrm{v}}\left[=108^{\mathrm{v}}\right]\right)$. Donc, de Ct 7,2 à 8,14, le Rom., Coll. gr. 16 constitue un témoin partiel et indirect de la chaîne de Polychronios.

58 Par conséquent, le manuscrit est un témoin complet de ce texte (qui se termine avec l'explication de Ct 6,9). Il n'est pas connu par Westerink (cité note 46). L'épilogue (= les versets 1202-1226 dans l'édition de Westerink) se trouve au f. 108 [= 109], où il parfait l'exégèse complète du Cantique dans le Rom., Coll. gr. 16.

59 Une telle chaîne, qui se compose de fragments de celle de Polychronios et de la catena Hauniensis, sans y ajouter d'autres extraits, est aussi identifiée pour l'Ecclésiaste. Labate a découvert que ce même manuscrit Rom., Coll. gr. 16 contient deux chaînes semblables sur l'Ecclésiaste: aux f. $25^{\mathrm{v}}-31^{\mathrm{v}}$ (aussi présente aux f. 235-242 du Romanus, Collegii graeci 9, manuscrit du $14^{\mathrm{e}}$ siècle: ces folios pourraient être une copie de ceux du Coll. gr. 16, d'après Labate) et aux f. 60 $70^{v}$ (qui n'est pas la chaîne de Polychronios, comme Labate l'avait écrit précédemment). Voir Labate, Nuovi codici (cité note 23) 553; Labate, Nuove catene (cité note 23) 243-250; Catena Hauniensis in Ecclesiasten in qua saepe exegesis servatur Dionysii Alexandrini nunc primum edita ab A. LABATE. CCSG, 24. Turnhout/Leuven 1992, 35 et 215-253; et A. LABATE, Le catene sull'Ecclesiaste del cod. Collegio gr. 16, dans A. Di Benedetto Zimbone/F. Rizzo Nervo (éds.), Kavíoкıv. Studi in onore di Giuseppe Spadaro. Medioevo Romanzo e Orientale, 12. Soveria Mannelli 2002, 183-194. Il paraît possible d'identifier aussi une telle chaîne pour les Proverbes: tant le Rom., Coll. gr. 9 (f. 251ㄴ-259) que le Coll. gr. 16 (f. $1-25$ et $32-59^{v}$ ) contiennent des scholies sur ce livre biblique. Comme les traditions des chaînes sur les trois livres salomoniques paraissent vraiment semblables (cf. aussi notre note 52), il est fort possible que cette chaîne soit du même type que celles sur l'Ecclésiaste et sur le Cantique. 


\section{Oxoniensis, Bodleianus, Auctarii X.2.5.12 (D'Orville 414) ${ }^{60}$}

Le document qui est repris sous cette signature n'est pas un manuscrit: il se compose de deux livres imprimés de I. Meursius qui sont reliés dans un volume. Le premier livre est celui que nous avons évoqué plus haut (note 4), c'est-à-dire l'édition que Meursius a faite de trois textes exégétiques sur le Cantique: la chaîne du Ps.-Eusèbe, celle de Polychronios, l'In Canticum de Michel Psellos. Le deuxième est une édition de quelques autres fragments théologiques grecs réalisée par Meursius. ${ }^{61}$ Dans le D'Orville 414, des notes manuscrites sont ajoutées dans les marges de chacun de ces deux volumes. De plus, à deux endroits, quelques feuillets manuscrits sont insérés. Par conséquent, il est admis dans le catalogue des manuscrits qui se trouvaient dans la bibliothèque de Jacques Philippe D'Orville. ${ }^{62}$ D'après ce catalogue, toutes les notes marginales du D'Orville 414 de même que le texte aux folios insérés ont été écrits par Lukas Holste (Lucas Holstenius). Toutefois, nous n'avons rencontré ce nom nulle part dans le D'Orville 414, ni d'ailleurs aucun autre: l'annotateur ne s'est pas identifié. Il est sûr que les notes ont été écrites à Paris, comme la main anonyme l'a indiqué explicitement (voir la note du f. $10^{v}$ cité plus loin). Il n'est pas improbable de supposer que cette opération s'est passée avant 1680, l'année au cours de laquelle les manuscrits grecs de Jacques-Auguste de Thou (Jacobus Augustus Thuanus) ont été achetés par Jean-Baptiste Col-

60 Nous tenons à remercier J. De Landtsheer (Leuven), qui nous a signalé des références utiles, de même que I. De Vos (Oxford), qui nous a envoyé quelques photos du D'Orville 414 en avril 2011. Au mois d'août de la même année, nous l'avons consulté nous-mêmes sur place. Pour l'identification des pages/folios qui contiennent les fragments de la chaîne de Polychronios, voir le dernier paragraphe de cette section.

61 I. Meursi, Variorum divinorum liber unus. In quo auctores theologi graeci varii, antehac nunquam vulgati. Lugduni Batavorum 1619.

62 [T. GAISFORD], Codices manuscripti et impressi cum notis manuscriptis olim D'Orvilliani, qui in Bibliotheca Bodleiana apud Oxonienses adservantur. Oxonii 1806, 54. Le D'Orville 414 est donc un de ces «codices impressi cum notis manuscriptis» et non un codex manuscrit lui-même, comme il était renseigné erronément par $\mathrm{P}$. Moore, qui le comptait parmi les manuscrits du poème de Psellos sur le Cantique: voir P. Moore, Iter Psellianum. A Detailed Listing of Manuscript Sources for All Works Attributed to Michael Psellos, Including a Comprehensive Bibliography. Subsidia Mediaevalia, 26. Toronto 2005, 472 («this manuscript was copied from Meursius' printed edition of 1617»). 
bert, ${ }^{63}$ car à plusieurs reprises l'annotateur s'est encore référé à la bibliothèque de Thuanus (voir plus loin).

Plusieurs fois, l'annotateur a indiqué les sources auxquelles il a emprunté les informations transcrites dans les marges. Ces indications nous apprennent que pour le deuxième livre qui est relié dans le D'Orville 414, il a utilisé d'autres sources que pour le premier (c'est-à-dire l'édition des textes du Ps.-Eusèbe, de Polychronios et de Psellos préparée par Meursius), qui est le seul à nous intéresser.$^{64}$ Pour le troisième texte de ce livre, à savoir le poème exégétique de Psellos sur le Cantique, la source différait de celle employée pour les chaînes du Ps.-Eusèbe et de Polychronios. Dans le cas du texte psellien, le nombre de notices marginales est très petit: c'est seulement à la p. $122^{65}$ qu'on trouve quelques leçons variantes. L'annotateur n'a pas spécifié d'où il les a empruntées: à la page titre de ce texte (p. 113), il se réfère à une traduction latine ainsi qu'à un manuscrit grec, mais il ne les nomme pas explicitement comme étant ses sources. Donc le texte de Psellos peut être laissé de côté lorsqu'on examine le texte dans le D'Orville 414 qui est le sujet de cet article, la chaîne de Polychronios.

La raison pour laquelle le D'Orville 414 est cité dans cet article parmi les témoins manuscrits de la chaîne de Polychronios est d'une part le fait que les marges autour de la chaîne sont remplies de leçons variantes, d'additions, de commentaires sur la mise en page du manuscrit etc. ${ }^{66}$ et d'autre part l'observation que l'annotateur a copié de sa main la section complète de la chaîne sur $\mathrm{Ct}$ 4,4-7,5, qui est la section manquant dans l'édition de Meursius (voir plus haut). Entre les p. 102 (où se trouve la transition brutale dans l'exégèse de $\mathrm{Ct} 4,3 \mathrm{~cd}$ à celle du v. $7,2-5)^{67}$ et 103 ,

63 Cf. H. Omont, Inventaire sommaire des manuscrits grecs de la Bibliothèque nationale et des autres bibliothèques de Paris et des Départements. Paris 1898, xxx.

64 Dans le deuxième livre, l'annotateur fait mention de quelques codices Regii. Voir les notices dans les marges des p. 5 et 41 de Meursius, Variorum divinorum (cité note 61) dans le D'Orville 414. Nous constatons aussi que dans ce livre, les marginalia sont en général plus courts, moins nombreux et moins soignés que ceux qui accompagnent Meursius, Polychronii expositiones (cité note 4) dans le même D'Orville 414.

65 Les numéros de pages qui suivent sont ceux de Meursius, Polychronii expositiones (cité note 4) dans le D'Orville 414.

66 L'annotateur a aussi ajouté quelques observations dans les marges des notes que Meursius, Polychronii expositiones (cité note 4) 180-182 avait rédigées sur le texte de la chaîne de Polychronios.

67 L'annotateur a marqué cette transition dans le texte imprimé et l'a identifiée dans la marge. Il a répété cette observation (c'est-à-dire: il y a une grande lacune 
un ternion est inséré sur lequel il a copié cette section entière. ${ }^{68}$ Le texte qui se trouve sur ces 6 feuillets, tous écrits recto et verso, présente la forme d'une chaîne à pleine page, dans laquelle le texte biblique et l'exégèse sont séparés l'un de l'autre avec les mots (presque toujours

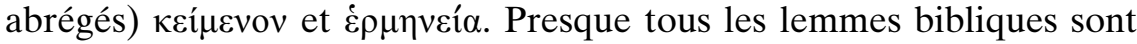
abrégés: la partie centrale du lemme est remplacée par «etc.».

La source à laquelle l'annotateur a emprunté ces additions assez extensives sur la chaîne de Polychronios est nommée plusieurs fois. Dans une notice à la page titre de cette chaîne, on lit que le texte de Meursius a été complété sur base d'un manuscrit de la bibliothèque de Thuanus: «Suppletus est Polychronii commentarius ex ms. codice biblioth[ecae] Ampliss[imi] D[omi]n[i] Thuani» (p. 75). ${ }^{69}$ Que cette notice ne se rapporte pas seulement aux marginalia, mais aussi à la section de Ct 4,4-7,5 copiée dans le ternion inséré, est démontré par une notule dans la marge supérieure du premier folio (f. 5) de ce ternion, où l'annotateur a confirmé qu'il a pris aussi cette section extensive de la chaîne du manuscrit de Thuanus («Supplementum expositionis Polychronii in canticum canticorum ex editione V[iri] Cl[arissimi] Joannis Meursii pag. 102 v. 8 ex Ms. codice bibliothecae Cl[arissimi] et Amplissimi V[iri] J. A. Thuani»). Probablement s'agit-il du même manuscrit auquel il a emprunté aussi les notes marginales qui précèdent et suivent cette section insérée, comme on peut le déduire de la lecture d'une note à la fin du dernier folio (f. $10^{v}$ ) du ternion, qui prouve un lien entre le texte dans ce ternion et les notes marginales («et quae sequuntur in edito. Haec ex Ms. Ampl[issimi] D[omi]n [i] Thuani descripsi Lutetiae Parisiorum»).

Tout cela nous incite à conclure que l'annotateur a copié toutes les leçons variantes, additions etc. de la chaîne de Polychronios sur base d'un seul manuscrit qui se trouvait dans la bibliothèque de Thuanus à Paris. En effet, entre les témoins manuscrits connus de cette chaîne,

dans le texte à la p. 102) dans la marge de la p. 182, dans la section portant sur la chaîne de Polychronios dans les notes de Meursius, Polychronii expositiones (cité note 4).

68 Le ternion n'est pas folioté. Nous utilisons ici le foliotage fictif $5-10^{\mathrm{v}}$ en nous référant aux feuillets en question. Comparez avec notre note 72 .

69 Pour une étude récente sur la bibliothèque de Thuanus, voir I. A. R. DE SMET, Thuanus. The Making of Jacques-Auguste de Thou (1553-1617). Travaux d'Humanisme et Renaissance, 418. Genève 2006, 171-191 (où le lecteur prendra aussi connaissance de références bibliographiques complémentaires). 
deux ont appartenu à Thuanus: les Parisini gr. 173 et $948 .{ }^{70}$ Sans doute, c'est à l'un de ces deux manuscrits que l'annotateur du D'Orville 414 a emprunté les morceaux de la chaîne de Polychronios. Cette conclusion est renforcée par un bref examen des matériaux dans les marges du premier texte dans le D'Orville 414, la chaîne du Ps.-Eusèbe.

Comme la chaîne de Polychronios, celle du Ps.-Eusèbe sur le Cantique est accompagnée dans le D'Orville 414 par des matériaux manuscrits assez nombreux et extensifs. Et également pour cette dernière chaîne (comme pour celle de Polychronios), l'annotateur a puisé à un manuscrit qui appartenait à Thuanus, comme il l'indique à la page de titre de la chaîne du Ps.-Eusèbe (p. 1): «Commentariolus qui in margine descriptus est habetur sub nomine Eusebii Pamphili in bibliotheca Ampl [issimi] D[omi]n[i] Thuani, unde eum descripsi». Les marginalia en question se composent de deux types: d'une part, on trouve des leçons variantes individuelles, et d'autre part des fragments exégétiques plus longs (portant sur Ct 1,1-6,9) ${ }^{71}$ dont chacun est lié par un index au verset biblique qu'il commente. Ces fragments exégétiques manuscrits sont continués (pour Ct 6,10-8,14) sur un binion qui est inséré entre les p. 58 et 59 de l'édition. ${ }^{72}$ Sur ces folios ajoutés, le commentaire possède la structure suivante: quelques mots du verset biblique (ou le numéro du verset) sont cités et suivis par le fragment correspondant.

La note citée plus haut nous informe que tous ces fragments que l'annotateur a ajoutés à la chaîne du Ps.-Eusèbe (ceux dans les marges autant que ceux dans le binion inséré) proviennent d'un manuscrit où ils

70 Cf. H. Omont, Inventaire sommaire des manuscrits grecs de la Bibliothèque nationale. Première partie. Ancien fonds grec: théologie. Paris 1886 (= première partie de son Inventaire [cité note 63]), 10 et 182. Voir aussi le catalogue originel de la bibliothèque de Thou: Catalogus bibliothecae Thuanae a clarissimis viris $\mathrm{P}$. et J. Puteanis ordine alphabetico primum distributus; tum a clarissimo viro I. Bullialdo secundum scientias et artes digestus; denique editus a J. Quesnell Parisino, et bibliothecario, cum indice alphabetico auctorum. Parisiis 1679 (nous avons consulté la reproduction Hamburgi 1704), II, 437 («Cantica Canticorum cum Scholiis Eusebii Pamphili et Polychronii Diaconi»= Paris. gr. 948) et 438 («Catena Graecorum Patrum in Cantica Canticorum, Eusebius in Cantica Canticorum»= Paris. gr. 173).

71 Dans l'édition de Meursius (cité note 4), la chaîne du Ps.-Eusèbe se termine par l'explication de Ct 6,9.

72 Le binion n'est pas folioté. Nous utilisons ici le foliotage fictif $1-4^{\mathrm{v}}$ en nous référant aux feuillets en question. L'annotateur a seulement écrit sur les f. $1-3^{\mathrm{v}}$; les f. $4-4^{\mathrm{v}}$ sont blancs. La raison justifiant la position du binion ajouté (c'est-àdire entre les p. 58-59) ne nous apparaît pas clairement: il se trouve au milieu d'un fragment dans la chaîne imprimée sur Ct 5,7 qui est attribué (pas tout à fait correctement) à Philon (de Carpasie). 
sont attribués à Eusèbe de Pamphile. Toutefois, aucun de ces fragments ne se trouve dans la chaîne du Ps.-Eusèbe (ni dans l'édition de Meursius ni dans aucun manuscrit connu de cette chaîne). Bien que cette observation paraisse remarquable à première vue, la cause en est évidente: les fragments qui dans le D'Orville 414 sont ajoutés par l'annotateur ne font pas partie de la chaîne du Ps.-Eusèbe mais bien du commentaire (inédit) qui est attribué au même Père et qui a été découvert récemment par J.-M. Auwers (voir notre note 16). L'ensemble de tous les fragments ajoutés dans le D'Orville 414 correspond au texte complet de ce commentaire. ${ }^{73}$ De ce commentaire, on ne connaît que deux manuscrits: ce sont précisément les Parisini gr. 173 et 948, qui ont appartenu à Thuanus et pour lesquels nous avons conclu précédemment que l'un d'entre eux a servi comme la source à laquelle l'annotateur du D'Orville 414 a puisé les matériaux de la chaîne de Polychronios. Il n'y a donc pas lieu de supposer que l'annotateur n'aurait pas consulté une même source pour ces deux textes: la chaîne de Polychronios et le commentaire (à distinguer de la chaîne) attribué à Eusèbe. La source est soit le Parisinus gr. 173 soit le 948.

Pour récapituler, le D'Orville 414 contient dans les marges des p. $75-$ $112^{74}$ des leçons variantes, des additions etc. de la chaîne de Polychronios, et surtout les f. $5-10^{\mathrm{v}}$ qui sont insérés entre les p. 102-103 contiennent une copie manuscrite de la section de cette chaîne portant sur Ct 4,4-7,5. Ces fragments sont copiés du Parisinus gr. 173 ou $948,{ }^{75}$ certainement après 1617 et peut-être avant 1680 .

73 Aucun des fragments écrits dans les marges ou dans le binion ne correspond à la chaîne du Ps.-Eusèbe. De plus, aucune des leçons variantes offertes dans les marges ne se rapporte à cette chaîne: elles sont toutes des variantes du texte biblique. On peut donc en conclure que l'annotateur n'a consulté aucun manuscrit de la chaîne du Ps.-Eusèbe (mais seulement un du commentaire attribué à Eusèbe).

74 C'est-à-dire du premier livre de Meursius qui est relié dans le D'Orville 414.

75 Nous ne nous risquerons pas à nous prononcer sur la question: lequel de ces deux codex parisiens est la source?, car nous n'en avons vu qu'un (le Parisinus gr. 173). Nous sommes d'avis qu'un examen des deux manuscrits s'avère nécessaire, présumant qu'ils sont très proches l'un de l'autre. D'après les renseignements donnés par Omont (voir notre note 70), ils sont tous deux écrits par Andréas Darmarios et leur contenu est presque totalement identique: ils contiennent la chaîne de Polychronios de même que le commentaire attribué à Eusèbe - seulement l'ordre est différent. En conséquence, ils sont probablement copiés à partir de la même source ou l'un sur base de l'autre. Nous nous limitons à observer que le D'Orville 414 et le Parisinus gr. 173 contiennent tous deux la même rédaction de la chaîne de Polychronios et sont donc très proches l'un de l'autre - cette observation est confirmée par un examen du commentaire du Ps.- 
Nous espérons que ces témoins inconnus, que nous avons mis en évidence, peuvent contribuer à l'établissement d'une édition critique de la chaîne de Polychronios sur le Cantique ou au moins être utiles pour l'évaluation de la popularité dont cette compilation a joui $\mathrm{du} 12^{\mathrm{e}}$ au $17^{\mathrm{e}}$ siècle.

\section{Annexe: nouveaux témoins manuscrits des prologues de la chaîne de Polychronios sur le Cantique}

Dans les manuscrits que nous avons énumérés ci-dessus, la chaîne de Polychronios sur le Cantique s'ouvre avec deux prologues composés. ${ }^{76}$

(1) Le premier prologue se compose de deux parties: (1a) l'intro-

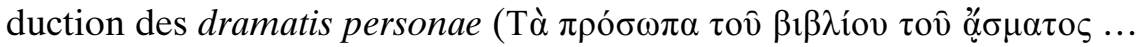

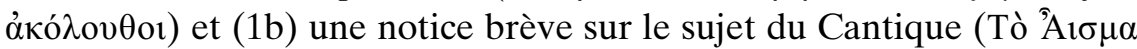

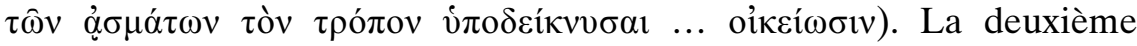
partie de ce premier prologue est caractéristique de la chaîne de Polychronios: elle ne se trouve dans aucune autre chaîne sur le Cantique. La première partie se rencontre aussi en appendice de l'Épitomé de Procope de Gaza sur le Cantique, mais dans une rédaction différente (et pas dans tous les manuscrits de cet épitomé). ${ }^{77}$ La permière partie est imputable à Origène; la deuxième à Basile de Césarée. ${ }^{78}$

Eusèbe dans ces deux témoins. D'autre part, nous avons détecté un bon nombre de petites différences entre le D'Orville 414 et le Parisinus gr. 173: pourraientelles être un indice que c'est le Parisinus gr. 948 qui a servi comme source à l'annotateur?

76 Récemment, tous ces prologues ont été édités (sans apparat critique) par $\mathrm{Au}-$ wers: voir son L'interprétation (cité note 16) Annexes, 32-36. Bien que cette édition soit celle que nous citons, nous informons nos lecteurs que tous ces prologues se trouvent aussi dans Meursius, Polychronii expositiones (cité note 4) $77-79$.

77 Cet épitomé ( $C P G$ 7431) occupe une position centrale dans la tradition des collections exégétiques sur le Cantique. Nous l'avons consulté dans l'édition préparée par J.-M. Auwers, Procopii Gazaei Epitome in Canticum canticorum. Editio critica, Dissertation présentée pour l'obtention du grade d'Agrégé de l'Enseignement Supérieur. Louvain-la-Neuve 2007 (à paraître dans la CCSG. Nous remercions vivement le Prof. Auwers pour nous avoir envoyé une copie de son édition préliminaire). Cet appendice ne figure pas dans la $P G 87^{2}, 1545-$ 1780.

78 Voir M. Harl, Les trois livres de Salomon et les trois parties de la philosophie dans les Prologues des Commentaires sur le Cantique des Cantiques (d'Origène aux Chaînes exégétiques grecques), dans J. Dummer et al. (éds.), Texte und 
(2) Le deuxième prologue fournit une explication du titre ${ }^{\top} A \imath \mu \alpha$ Q̊㇒ $\mu_{\alpha} \tau \omega v .{ }^{79}$ Comme l'avait déjà remarqué Faulhaber, ce deuxième prologue se compose de trois parties, ${ }^{80}$ dont la première (2a: ${ }^{\wedge} \mathrm{A} 1 \sigma \mu \alpha$

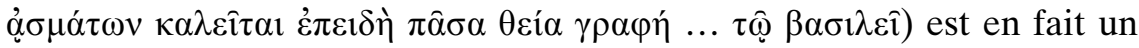
résumé des deux premiers prologues d'une autre chaîne sur le Cantique,

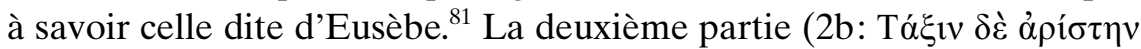

Textkritik. Eine Aufsatzsammlung. TU, 133. Berlin 1987, 265-266 et Auwers, L'interprétation (cité note 16) II, 437 et 447.

79 En fait ce «deuxième prologue» n'est pas un véritable prologue, mais le commentaire du titre du Cantique. Voir aussi Auwers, L'interprétation (cité note 16) II, 410.

80 Faulhaber (cité note 3) 44-45.

81 La chaîne du Ps.-Eusèbe s'ouvre par quatre prologues, qui ont tous leur propre titre. Une excellente étude récente de tous ces prologues est à lire chez AuwERs, L'interprétation (cité note 16) II, 411-449. Les textes de ces prologues peuvent être consultés aux pages 16-28 des annexes du même ouvrage et dans MeurSIUS, Polychronii expositiones (cité note 4) 3-12. Nous signalons que HarL (cité note 78) 261 note 55 et 268 a découvert que le premier prologue est emprunté à la synopse du Cantique que donne le Ps.-Athanase ( $C P G$ 2249; cf. $P G$ 28, 349C-357B). Plus tard, Auwers, L'interprétation (cité note 16) II, 411-424 a étudié le lien entre ces deux textes avec davantage de détails. Il a aussi inclus une discussion du rapport avec la synopse du Vaticanus, Barberinianus gr. 317 (s. XI), f. $174^{\mathrm{v}}-184$, qui est aussi apparentée à celle du Ps.-Athanase, comme on l'avait déjà remarqué précédemment; voir G. Dorival, Le document synoptique du Barberinianus gr. 317 (III 36), dans G. Dorival et al. (éds.), Qu'est-ce qu'un corpus littéraire? Recherches sur le corpus biblique et les corpus patristiques. Collection de la Revue des Études juives, 35. Paris/Louvain/Dudley, MA 2005, 95-108. À notre avis, deux autres documents devraient également être étudiés: la synopse du Cantique du Ps.-Chrysostome ( $C P G$ 4559), inédite et préservée dans le Neapolitanus, Bibliothecae Nationalis II.A.12 (s. XIV), f. $63-68^{\mathrm{V}}$ et la synopse de Nicétas Seides. Sur le manuscrit italien, voir P. DE Lagarde, Septuaginta-Studien. IV. Eine neue Recension der Septuaginta. Abhandlungen der königlichen Gesellschaft der Wissenschaften zu Göttingen 38 (1892) 59-102 et G. Dorival, L'apport des Synopses transmises sous le nom d'Athanase et de Jean Chrysostome à la question du corpus littéraire de la Bible, dans Dorival et al. (éds.), Corpus littéraire, 55-70. La synopse de Nicétas, un auteur constantino-

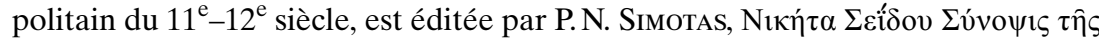

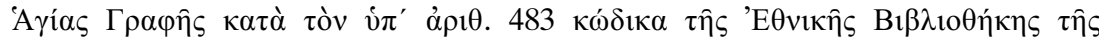

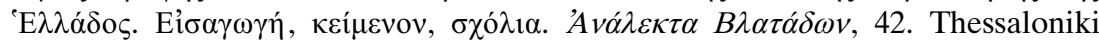
1984, 252-257 d'après un seul manuscrit. La part concernant le Cantique se trouve aussi dans l'Athous, Iviron 267 (s. XVI), f. 254-257 et dans l'Athous, Scetae sancti Prodromi 4 (a. 1692), f. 179-181v. Dans son introduction (32-41), Simotas a indiqué la ressemblance très forte entre les synopses du Cantique que donnent Nicétas et le Ps.-Athanase. Toutefois, nous avons identifié aussi des accords entre le texte de Nicétas et ceux du Barberinianus gr. 317 et du premier prologue du Ps.-Eusèbe, contre le Ps.-Athanase. Une étude détaillée avec une 


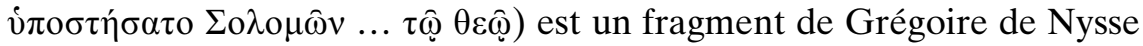
qui se retrouve dans une rédaction semblable dans l'Épitomé de Procope $^{82}$ et dans le deuxième prologue de la chaîne dite d'Eusèbe. ${ }^{83}$ De

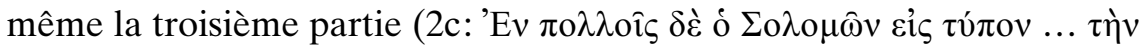
$\delta v ́ v \alpha \mu \imath v)$ est-elle tirée de Grégoire de Nysse; elle se retrouve presque identiquement dans le troisième prologue de la chaîne dite d'Eusèbe ${ }^{84}$ et dans le prologue à la catena Barberiniana. ${ }^{85}$

Comme on le constate, la presque totalité de ces deux prologues et leurs différentes parties se retrouvent sous une forme plus ou moins semblable dans les chaînes apparentées. ${ }^{86}$ Néanmoins, on peut identifier les éléments de ces pièces introductives qui sont caractéristiques de la chaîne de Polychronios. Par exemple, la partie 1 b se retrouve seulement dans cette chaîne et la rédaction des prologues $1 \mathrm{a}, 2 \mathrm{a}$ et $2 \mathrm{~b}$ peut aisément être distinguée de celle présente dans la chaîne du Ps.-Eusèbe et dans l'Épitomé de Procope. En outre, la séquence des parties différentes des prologues qu'on peut lire dans la chaîne de Polychronios est unique.

comparaison de l'ensemble de ces quatre textes s'avère nécessaire. Nous signalons enfin que Harl (cité note 78) 261 note 56 et Auwers, L'interprétation (cité note 16) II, 420 note 27 ont indiqué que dans le Parisinus gr. 151, la chaîne de Polychronios est précédée par une synopse, qu'ils ont identifiée comme celle du Ps.-Athanase. Néanmoins, comme Harl a ajouté que ce texte est un peu retouché, on pourrait se demander s'il est la synopse de Nicétas, celle du Ps.Athanase voire une autre. Cette question montre que le texte de Nicétas doit être inclus dans la discussion. Voir, en outre, notre note 98.

82 Auwers, Procopii Epitome (cité note 77), no. 3. Ce fragment se trouve aussi dans la $P G 87^{2}, 1545 \mathrm{~B}-1547 \mathrm{~A}$.

83 Voir Auwers, L'interprétation (cité note 16) Annexes, 26 ou Meursius, Polychronii expositiones (cité note 4) 10.

84 Voir Auwers, L'interprétation (cité note 16) Annexes, 28 ou Meursius, Polychronii expositiones (cité note 4) 11.

85 Auwers, L'interprétation (cité note 16) Annexes, 38. «Catena Barberiniana» est le nom qu'on donne à la chaîne du Vaticanus, Barberinianus gr. 388 (s. XIII), f. 130-162 . Sans doute, cette compilation est-elle apparentée à l'Épitomé de Procope, mais aucun accord ne se dégage sur les modalités de ce lien. Voir FAulhaber (cité note 3) 37-39; Barbàra, Origene (cité note 8) 117-122; J.-M. Auwers, Du bon usage d'une édition d'Origène. À propos du Commentario al Cantico dei cantici de M. A. Barbàra. Adamantius 13 (2007) 287-296; M. A. BARBÀra, La catena sul Cantico dei cantici trasmessa dal codice Barberiniano gr. 388. Adamantius 14 (2008) 329-351; Auwers, Procopii Epitome (cité note 77) LXI-LXXII.

86 Voir les tableaux et les discussions dans Harl (cité note 78) 263-269 et surtout Auwers, L'interprétation (cité note 16) II, 437-439 et 444-449. 
Ces prologues ne sont pas seulement transmis dans la tradition de la chaîne de Polychronios, dont nous avons énuméré ci-avant les témoins. Ils se rencontrent également, complets ou partiels, dans quelques autres manuscrits, qui ne sont pas des témoins de la chaîne de Polychronios, mais des autres genres des chaînes ou commentaires sur le Cantique qui ont toutefois pris ces prologues de la chaîne de Polychronios.

Jusqu'à présent, un seul manuscrit (qui n'est donc pas un témoin de la chaîne de Polychronios, mais qui contient tout de même une partie de ses prologues) est connu. C'est un manuscrit de la main d'Andréas Darmarios qui transmet la chaîne du Ps.-Eusèbe et celle dite des Trois Pères ( $C P G \mathrm{C} 81$ type $\mathrm{B} 2) .{ }^{87} \mathrm{La}$ présence du fragment d'un des prologues de Polychronios dans ce manuscrit a été signalée par Auwers:

\section{Salmanticensis, Bibliothecae Universitatis 2716 (olim Matritensis, Pa-} latinus 20) (s. XVI), f. $6^{\mathrm{v} 8}$

Les f. $1-48^{v}$ de ce manuscrit contiennent le texte de la chaîne dite d'Eusèbe. Grâce aux recherches d'Auwers, on sait que ce manuscrit ajoute un cinquième prologue aux quatre par lesquels la chaîne du Ps.Eusèbe s'ouvre dans ses autres manuscrits. ${ }^{89}$ Comme Auwers l'avait remarqué, ce cinquième prologue est en fait la première partie du

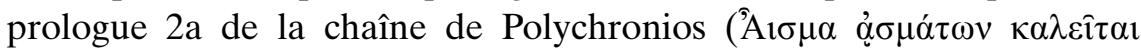

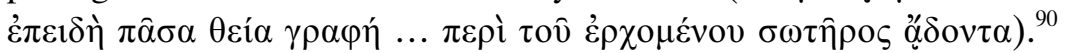

À ce manuscrit, nous pouvons en ajouter trois autres, au sein desquels on retrouve les prologues, complets ou partiels, de la chaîne de Polychronios sur le Cantique:

2. Athous, Iviron 329 (s. XVI), f. $175-175^{\mathrm{v}}$

87 En fait, dans ce manuscrit, une des trois sources de la chaîne des Trois Pères (l'In Canticum de Michel Psellos, f. 49-78) est séparée des deux autres (le commentaire au Cantique de Théodoret de Cyr [CPG 6203] et celui dit des Trois Pères [CPG C 81 type B1], f. 80-229). Voir déjà Faulhaber (cité note 3) 7-8.

88 Voir Ch. Graux/A. Martin, Rapport sur une mission en Espagne et en Portugal. Notices sommaires des manuscrits grecs d'Espagne et de Portugal. Nouvelles archives des missions scientifiques et littéraires 2 (1892) 86-89 et T. SANTANDER, La Biblioteca de don Diego de Covarrubias y Leyva, obispo de Ciudad Rodrigo y de Segovia, y Presidente del Consejo de Estado (1512-1577). I. Manuscritos. Salamanca 2000, 83-85.

89 Auwers, L'interprétation (cité note 16) II, 436 et 450 note 4. Sur les quatre prologues de la chaîne du Ps.-Eusèbe, voir notre note 81 .

90 Auwers, L'interprétation (cité note 16) II, 436. 
Aux f. $175^{\mathrm{v}}-176$ de ce manuscrit figure une partie de l'In Canticum de Psellos. ${ }^{91}$ Elle est précédée par un bref fragment anonyme, que Spyridon Lambros a décrit vaguement comme un extrait «sur le Cantique». ${ }^{92}$ Ayant consulté nous-mêmes ce fragment, nous sommes en mesure de l'identifier comme les deux prologues complets de la chaîne de Polychronios (c'est-à-dire: prologues $1 \mathrm{a}-1 \mathrm{~b}-2 \mathrm{a}-2 \mathrm{~b}-2 \mathrm{c}) .^{93}$

\section{Vindobonensis, theologicus gr. 258 (s. XVI), f. 35}

Ce manuscrit contient la chaîne dite d'Eusèbe sur le Cantique (f. 1 $34^{v}$ ) et l'In Canticum de Psellos (f. 36-68). D'après le catalogue de Herbert Hunger et al., ce dernier texte est précédé au f. 35 par un extrait anonyme, qui est décrit comme des prolégomènes au texte de Psellos. ${ }^{94}$ Bien que nous n'ayons pas vu le manuscrit, les premiers et derniers

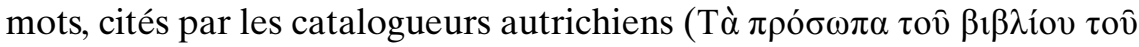

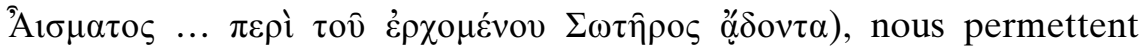

91 En fait, ce manuscrit est l'un des meilleurs témoins de ce texte: voir WESTERINK (cité note 46) $\mathrm{x}$.

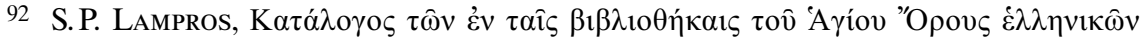

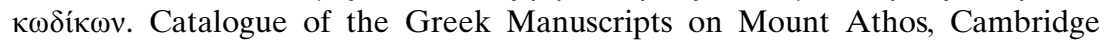
1900, II, 86. Nous avons consulté des photos du manuscrit à l'IRHT.

93 Entre les prologues $1 \mathrm{~b}$ et $2 \mathrm{a}$, ce manuscrit contient la phrase suivante, qui est

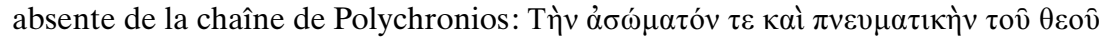

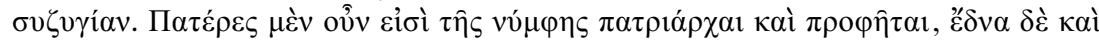

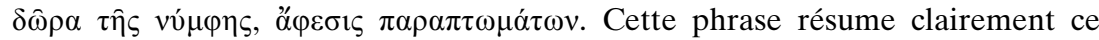
qu'avait écrit Grégoire de Nysse dans sa première homélie sur le Cantique ( $C P G$ 3158): voir son texte complet dans LANGERBECK (cité note 18) 15, 1. 13-15 et 24, 1. 2-6. La seule autre compilation dans laquelle nous l'avons trouvée, est le résumé du début de la catena Hauniensis qui se trouve aux f. $70^{\mathrm{v}}-71$ du Rom., Coll. gr. 16 (cf. au-dessus). En effet, la phrase telle qu'elle se lit dans l'Iviron 329 se retrouve dans la marge supérieure du f. $71 \mathrm{du}$ manuscrit romain, au-dessus du début du prologue 2a de la chaîne de Polychronios. De plus, l'accusatif avec lequel s'ouvre la phrase dans l'Iviron 329 est absurde dans ce manuscrit, mais dans le Coll. gr. 16 il suit la préposition $\pi \rho o ́ \varsigma$, qui est le dernier mot de la marge

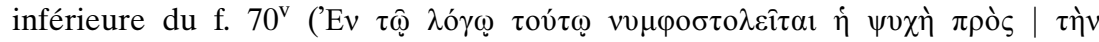
$\grave{\alpha} \sigma \omega ́ \mu \alpha \tau o ́ v \kappa \tau \lambda$.). En d'autres termes, à première vue, il nous semble assez probable que, dans le manuscrit athonite, l'extrait du résumé de la catena Hauniensis est inséré dans les prologues de la chaîne de Polychronios, alors que ces deux éléments sont clairement séparés l'un de l'autre dans le Coll. gr. 16. En conséquence, il est possible qu'en transmettant le texte des prologues de la chaîne de Polychronios, l'Iviron 329 dépende du Romanus, Coll. gr. 16.

94 H. Hunger/W. Lackner unter Mitarbeit von CH. Hannick, Katalog der griechischen Handschriften der Österreichischen Nationalbibliothek. Teil 3/3: Codices theologici 201-337. Museion. Veröffentlichungen der Österreichischen Nationalbibliothek, Neue Folge. Vierte Reihe: Veröffentlichungen der Handschriftensammlung, 1.3.3. Wien 1992, 197-199. 
d'identifier ces prolégomènes anonymes. L'incipit est celui du prologue 1a de la chaîne de Polychronios. Le desinit figure dans le début du prologue $2 \mathrm{a} .{ }^{95}$ Par conséquent, on peut supposer que le texte de Psellos est précédé par le prologue 1a (peut-être suivi par 1b, mais sans certitude) et le début du 2a de la chaîne de Polychronios.

\section{Hierosolymitanus, Sancti Sepulchri 370 (s. XVI), f. 312-312v}

Les f. $312^{\mathrm{v}}-316^{\mathrm{v}}$ de ce manuscrit contiennent le texte septantique du Cantique avec des scholies anonymes dans les marges. ${ }^{96}$ Il est précédé par un prologue anonyme (f. $310^{\mathrm{v}}-312$ ). ${ }^{97}$ Nous avons identifié ce prologue de la façon suivante: il se compose d'une synopse du Cantique qui est liée à celles du Ps.-Athanase et de Nicétas ${ }^{98}$ et d'une rédaction abrégée et confuse de quelques prologues de la chaîne de Polychronios: 1a, 2b, 2c (uniquement le début) et $1 \mathrm{~b} .{ }^{99}$ La plupart des citations bi-

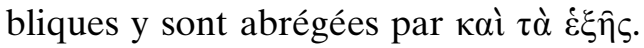

95 Vraisemblablement, la partie du prologue 2a qui se situe dans le Vindobonensis est celle qu'on peut lire dans le Salmanticensis 2716 (voir plus haut).

96 Pour l'Ecclésiaste aussi, ce manuscrit contient des scholies marginales (f. $305^{\mathrm{v}}-310^{\mathrm{v}}$ ). D'après Labate, Nuove catene (cité note 23) 262, il s'agit d'un «breviatum» de la chaîne de Polychronios sur l'Ecclésiaste. Pour le Cantique, ce n'est pas le cas: bien que quelques scholies contiennent des parallèles verbaux avec la chaîne de Polychronios, nous avons trouvé un bon nombre d'éléments (des extraits du commentaire de Théodoret de Cyr) qui ne figurent nulle part dans la chaîne de Polychronios.

97 La description qu'a fournie Papadopoulos-Kérameus dans son catalogue est

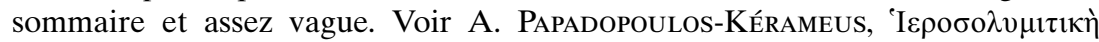

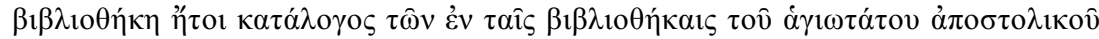

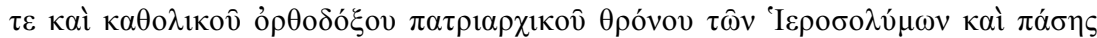

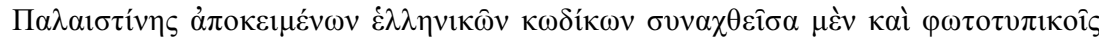

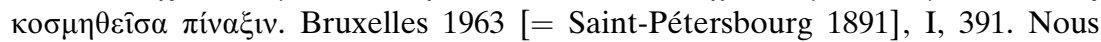
avons consulté des photos du manuscrit à l'IRHT.

98 Sur ces synopses, cf. plus haut (note 81). À première vue, la synopse du Sancti Sepulchri contient et des éléments en accord avec Nicétas contre le Ps.-Athanase, et d'autres qui sont identiques à la synopse du Ps.-Athanase, contre Nicétas. Par conséquent, elle trouverait sa place dans l'étude qui, à notre avis, devrait être menée (cf. note 81). Une combinaison d'une synopse et des prologues de la chaîne de Polychronios se rencontre aussi ailleurs, à savoir dans le Parisinus gr. 151.

99 Entre les prologues $2 \mathrm{~b}$ et $1 \mathrm{~b}$, le manuscrit livre la phrase suivante, qui est absente

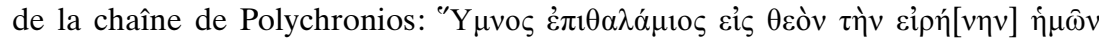

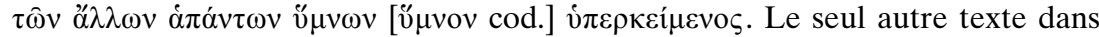
lequel nous avons trouvé une citation semblable est le prologue très court de l'anthologie du commentaire au Cantique de Théodoret de Cyr présent dans le Parisinus, Coislinianus 194 (nommé plus haut), f. 117-144, où il suit la chaîne de 


\begin{abstract}
Up to the present, 18 manuscript witnesses (excluding a few fragmentary ones) are known of the catena on the Song of Songs that is attributed to a certain Polychronius the Deacon and which has not yet been edited critically. In this article, several additional manuscripts are identified, which have been copied in the $12^{\text {th }}$ to $17^{\text {th }}$ centuries. Firstly, four codices are listed that contain the complete catena but have escaped the attention of previous scholarship. Secondly, two partial witnesses are identified: one of them is a printed book that contains handwritten sections of the catena and the other is a manuscript in which the catena of Polychronius is used as one of three sources that together form a new exegetical compilation. Thirdly and finally, three unknown manuscripts are presented that contain (parts of) the prologues to the catena of Polychronius, although they do not witness to the catena itself. In drawing attention to all of these manuscripts, the article aims to contribute to the project of a critical edition of this catena and to the appreciation of its transmission history and reception.
\end{abstract}

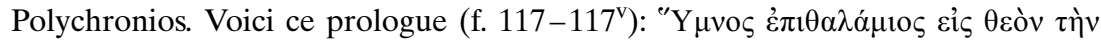

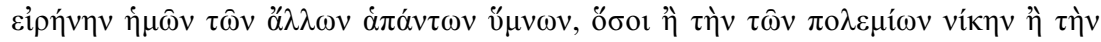

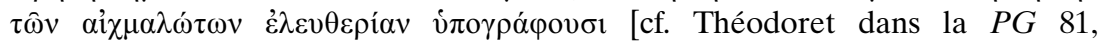

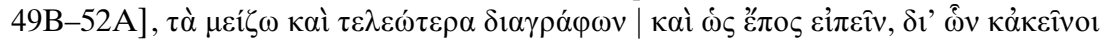

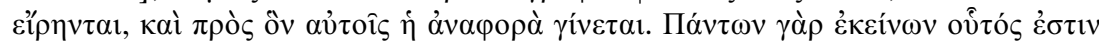

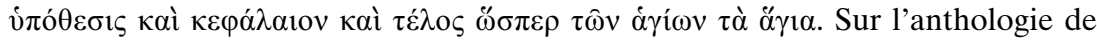
Théodoret dans ce manuscrit parisien, voir L. Bossina, Teodoreto e non Teodoreto. Storia di un equivoco lungo cinque secoli. Rivista di Storia del Cristianesimo 2 (2005) 199 et 206 (repris dans son Teodoreto restituito [cité note 3], 33 et 41). On devrait comparer toutes les scholies de Théodoret dans la chaîne du Sancti Sepulchri 370 avec le résumé qui se trouve dans le Coislinianus 194 et avec le commentaire de Théodoret tel qu'il est transmis dans la chaîne des Trois Pères (CPG C 81 type B2) et imprimé dans la $P G$ 81, 27-213 afin de voir si la rédaction de ces fragments dans le Sancti Sepulchri 370 est apparentée à celle du Coislinianus 194 (ce qui nous semble possible sur quelques points, mais pas sur tous). 